4 Sequestration of eosin dye by Magnesium (II) doped zinc oxide nanoparticles and its Kinetic, Isotherms and Thermodynamic

5 studies

6

Saruchi ${ }^{a *}$, Mayank Sharma ${ }^{b}$, Mohammad Rafe Hatshanc, Vaneet Kumard and Ashvinder Rana 8

$\mathbf{a}^{*}$ Department of Biotechnology, CT Institute of Pharmaceutical Sciences, Jalandhar, Punjab, India

bepartment of Biotechnology, DAV University, Jalandhar

$11{ }^{\mathrm{c} C h e m i s t r y}$ Department, College of Science, King Saud University, Riyadh 11451, Saudi Arabia

${ }^{\mathrm{d}}$ Department of Applied Science, CT Group of Institutions, Shahpur Campus, Jalandhar, Punjab, India

Corresponding Author:

15 Dr Saruchi

16 Associate Professor

17 Department of Biotechnology

18 CT Group of Institutions, Shahpur Campus, Jalandhar, Punjab, India

19 suruchinitj15@gmail.com

20

21 
23 Table S1: Effect of adsorbent dose, agitation speed, dye concentration, pH and temperature on eosin adsorption onto 24 synthesized $\mathrm{ZnO}$ and $\mathrm{Mg}^{+2}$ doped $\mathrm{ZnO}$ nanoparticles

25
Parameters
ZnO NPs
$\mathrm{Mg}^{+2} \mathrm{ZnO}$ NPs

Maximum Removal (\%) Standard Deviation

Maximum Removal (\%)

Standard Deviation

Adsorbent dose (g)

$\begin{array}{lcc}0.1 & 31.6 & 0.948 \\ 0.2 & 59.2 & 1.776 \\ 0.3 & 74.3 & 2.229 \\ 0.4 & 89.4 & 2.682 \\ 0.5 & 97 & 2.91 \\ 0.6 & 97.2 & 2.916\end{array}$

32.9

0.987

61.7

1.851

78.2

2.346

91.3

2.739

98.2

2.946

98.3

2.949

Agitation (rpm)

50

75

100

125
49.4

58.6

69.5

81.4
1.482

1.758

2.085

2.442
51.3

1.539

62.5

72.4

83.6
1.875

2.172

2.508 
150

175

200

\section{Dye Concentration}

(ppm)

10
20
30
40
50
pH
2
4
6
7
8
10

96.8

92.3

89.4

97.3

89.6

77.8

68.4

54.6

30.7

65.6

97.2

96.5

63.5

45.6
2.904

2.769

2.682

2.919

2.688

2.334

2.052

1.638

0.921

1.968

2.916

2.895

1.905

1.368
97.9

94.2

92.1

2.763

98.1

2.943

91.7

81.4

73.2

59.4

2.751

2.442

2.196

1.782

71.2

98.2

97.1

68.4

44.9
1.182

2.136

2.946

2.913

2.052

1.347 
Temperature $\left({ }^{\circ} \mathrm{C}\right)$

25

35

45

55
54.3

76.7

89.4

97.3
1.086

1.534

1.788

1.946
56.9

79.3

90.6

98.2
1.138

1.586

1.812

1.964 
Table S2: Langmuir, Freundlich, Temkin and Dubinin-Radushkevich (D-R) isotherm for the adsorption of eosin onto synthesized $\mathrm{ZnO}$ and $\mathrm{Mg}^{+2} \mathrm{ZnO}$ nanoparticles

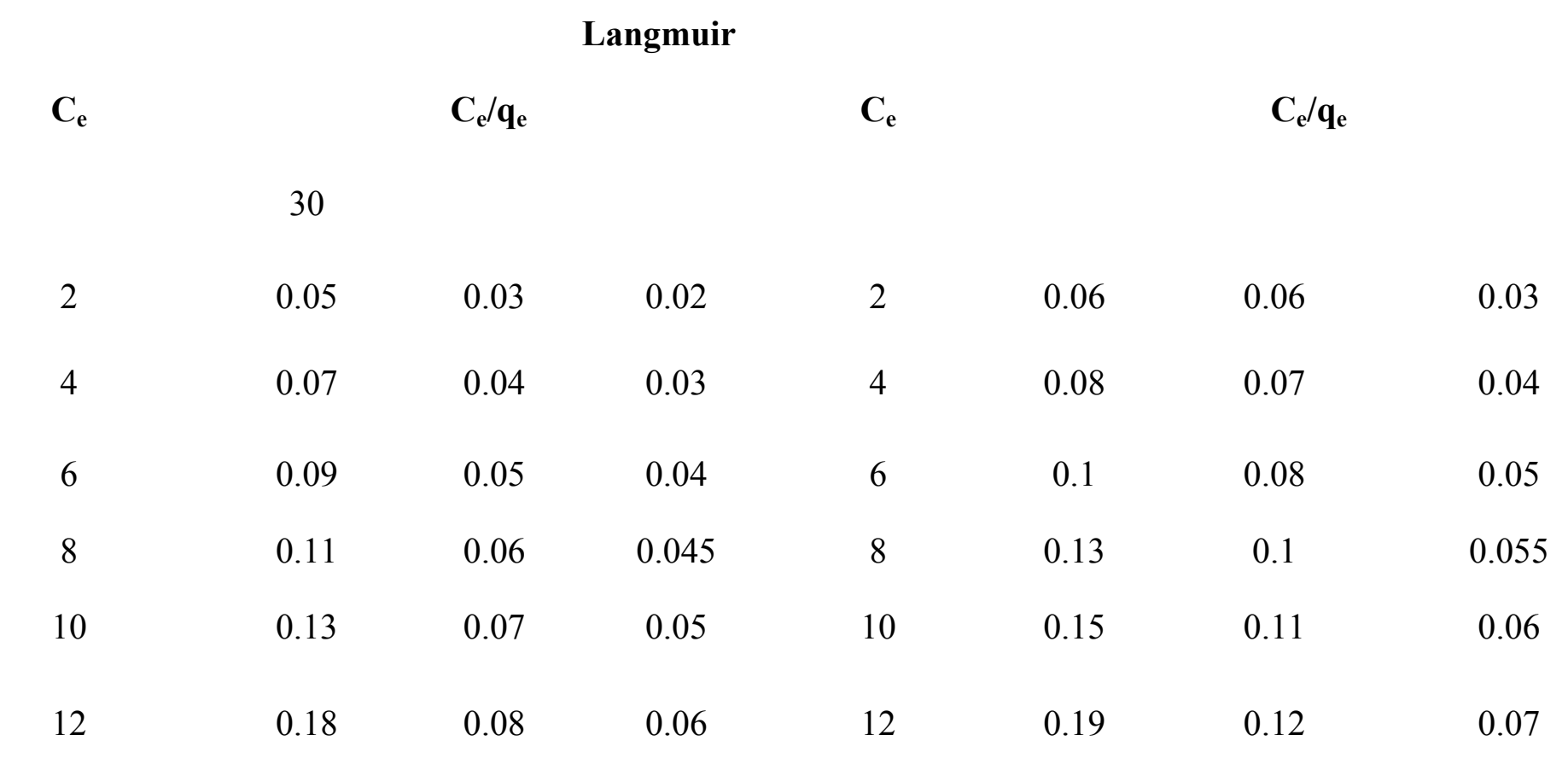

\section{Freundlich}

$\log \mathbf{C}_{\mathrm{e}}$

$\log q_{e}$

$\log \mathrm{C}_{\mathrm{e}}$

$\log q_{e}$ 


$\begin{array}{cccccccc}0.2 & 0.9 & 1.1 & 1.3 & -2.5 & 0.47 & 0.54 & 1.1 \\ 0.4 & 1.1 & 1.4 & 1.6 & -2 & 0.71 & 0.75 & 1.22 \\ 0.6 & 1.4 & 1.7 & 1.9 & -1.5 & 0.82 & 0.86 & 1.31 \\ 0.8 & 1.6 & 1.9 & 2.2 & -1 & 1.04 & 1.09 & 1.43 \\ 1 & 1.8 & 2.1 & 2.5 & 0 & 1.16 & 1.24 & 1.6 \\ 1.2 & 2 & 2.4 & 2.7 & 0.5 & 1.25 & 1.32 & 1.65 \\ 1.4 & 2.2 & 2.5 & 2.8 & 1 & 1.28 & 1.36 & 1.72\end{array}$

\section{Temkin}

$q_{e}$

$-3$

$-2$

$-1$

0

0.5

$\ln C_{e}$

$\mathbf{q}_{\mathrm{e}}$

$\ln \mathbf{C}_{\mathrm{e}}$

11.6

0.2

9

10

12

16.4

0.4

12

13

15

9.5

6.5

22.9

0.6

15

16

18

17.8

14.7

0.8

19

20

22

23.6

$20.6 \quad 34.1$

1

24

25

27

30.2

26.5

38

1.2

28

29

31 


$\begin{array}{rrrrrrrr}1.5 & 38.6 & 34 & 42 & 1.4 & 32 & 33 & 35 \\ 2 & 44 & 40.7 & 48 & 1.6 & 35 & 36 & 37\end{array}$

Dubinin-Radushkevich (D-R) isotherm

$\begin{array}{llll}\text { Temperature }\left({ }^{\circ} \mathrm{C}\right) & \mathbf{k}_{\mathrm{D}}\left(\mathrm{mol}^{2} \mathrm{~kJ}^{-2}\right) & \mathbf{E}\left(\mathrm{kJ} \mathrm{mol}^{-1}\right) & \mathrm{R}^{2}\end{array}$

\begin{tabular}{|c|c|c|c|c|c|c|}
\hline & ZnO NPs & $\mathrm{Mg}^{+2} \mathrm{ZnO}$ NPs & ZnO NPs & $\mathrm{Mg}^{+2} \mathrm{ZnO}$ NPs & ZnO NPs & $\mathrm{Mg}^{+2} \mathrm{ZnO}$ NPs \\
\hline 25 & 2.291 & 2.032 & 0.316 & 0.252 & 0.52 & 0.62 \\
\hline 50 & 2.971 & 2.912 & 0.289 & 0.241 & 0.55 & 0.58 \\
\hline 75 & 3.387 & 2.538 & 0.298 & 0.278 & 0.79 & 0.53 \\
\hline
\end{tabular}




\section{Optimization parameter}

Different reaction parameters like temperature, $\mathrm{pH}$, adsorbent dosage, dye concentration and agitation speed were optimized, so as to achieve maximum dye adsorption. It was clear from the results that maximum dye removal occurred at $\mathrm{pH}=6$, temperature $=55{ }^{\circ} \mathrm{C}$, adsorbent dosage $=0.6 \mathrm{~g}$, dye concentration $=10 \mathrm{ppm}$ and agitation speed $=150 \mathrm{rpm}($ Table $\mathrm{S} 1)$. The maximum dye removal was found to be $97.9 \%$ and $98.4 \%$ in $\mathrm{ZnO}$ NPs and $\mathrm{Mg}^{+2}$ doped $\mathrm{ZnO}$ NPs, respectively.

\section{Adsorption isotherms}

Langmuir isotherm showed that theoretical and experimentally adsorption capacity of eosin dye onto synthesized $\mathrm{ZnO}_{\mathrm{NPs}}$ and $\mathrm{Mg}^{+2}$ doped ZnO NPs is similar. $\mathrm{R}_{\mathrm{L}}$ value lies in the range of $0-1$, which indicated that adsorption mechanism is favourable. The $\mathrm{R}_{\mathrm{L}}$ value of dye adsorption is lower in $\mathrm{Mg}^{+2}$ doped $\mathrm{ZnO}$ NPs as compared to $\mathrm{ZnO}$ NPs, which showed strong interaction between eosin dye and $\mathrm{Mg}^{+2}$ doped $\mathrm{ZnO}$ NPs. Freundlich isotherm showed that $1 / \mathrm{n}$ value is more than 1 , this showed that it is favourable for adsorption condition. Temkin isotherm showed higher value of $b$, which indicated the strong interaction between eosin de and synthesized nanoparticles (Table S2). 